\title{
Modelling of macrosegregation with mesosegregates in a binary metallic cast by the diffuse approximate meshless method
}

\author{
Katarina Mramor ${ }^{1,2, a}$, Vanja Hatić ${ }^{1}$, Boštjan Mavrič ${ }^{1,2}$ and Božidar Šarler ${ }^{1,2}$ \\ ${ }^{1}$ Institute of Metals and Technology, Laboratory for Simulation of Materials and Processes, Lepi pot 11, 1000 Ljubljana, Slovenia \\ ${ }^{2}$ University of Ljubljana, Faculty for Mechanical Engineering, Laboratory for Fluid Dynamics and Thermodynamics, Aškerčeva cesta 6, 1000 \\ Ljubljana, Slovenia
}

\begin{abstract}
The solidification of binary metallic alloy $(\mathrm{Sn}-10 \% \mathrm{~Pb})$ is simulated in 2D with a diffuse approximate meshless method. The macrosegregation with mesosegregates is described by a coupled set of volume-averaged partial differential equations. The incompressible Newtonian fluid is described by coupled mass and momentum equations and the mushy zone is treated as a Darcy porous media. The energy transport is described with enthalpy formulation and the species transfer is incorporated by considering the Lever rule approximation. The thermo-solutal Boussinesq hypothesis is applied in order to account for the double-diffusive effects in the melt. The mathematical model is solved by a diffusive approximate method on local subdomains with Euler time stepping. Pressure-velocity coupling is incorporated by applying the fractional step method. The instabilities due to the convective terms are smoothed out by applying the upwinding technique. The results are presented on a regular node arrangement and are compared to other benchmark results. The present paper demonstrates that the results calculated with the diffuse approximate meshless method are in good agreement with reference results.
\end{abstract}

\section{Introduction}

Solidification is a phase change process that combines many different research areas ranging from continuum mechanics to thermodynamics. In recent years, the demand for materials with better structural quality and advanced physical properties resulted in steady raise in the research topic interest as the number of research articles has grown from 83 in 1970 to 3383 in 2017 [1]. The growth in the popularity of this research topic is mainly due to the requirements from industry, particularly those involving casting, as the solidification process plays an important role in the quality of the final cast product. By better understanding the physical phenomena during the solidification process, many defects, such as inhomogeneities can be either avoided or diminished. As experimenting with the casting of industrial size ingots can be often very expensive and time-consuming, the simulation programs can play an important role in the adjustment of the cast parameters. The simulation programs have to be thoroughly tested before they can be used for the application on an industrial project, especially when using novel numerical methods, such as meshless methods.

Among many varieties of meshless methods developed in recent years [2-6], the diffuse approximate method (DAM) [7] is chosen for the model presented in this paper. The method applies weighted least squares functions to determine a locally smooth and differentiable approximation of the discontinuous data in each particular computational point.

The DAM has already been applied to convective diffusive problems [8], natural convection in porous media [7], macrosegregation in columnar solidification [9], heat conduction and transport [10,11] problems. In the present paper, a benchmark test case devised for macrosegregation with mesosegragtes in binary metallic alloy by [12] was chosen and tested for $\mathrm{Sn}-10 \% \mathrm{~Pb}$.

The problem describes the solidification of binary alloy in a rectangular cavity. Numerically, it is described by a set of tightly coupled momentum, mass, energy and solute transport equations. The strong coupling and the transition from fully liquid to fully solidified state results in the formation of mesoscale channels- mesosegregates. The formation of mesosegergates has been previously observed for a similar alloy (Pb-18\%Sn), both experimentally [13] and numerically $[9,14]$. The present paper provides the results for $\mathrm{Sn}-10 \% \mathrm{~Pb}$ alloy and compares them to reference results calculated in [14].

\section{Model description and numerical solution}

\subsection{Problem description}

The present paper addresses a simplified solidification model previously described in $[12,14]$. The referenced

\footnotetext{
a Corresponding author: katarina.mramor@imt.si
} 
SMACS (Simulation of MACrosegregation and Structures project founded by the French Research Agency) benchmark solidification example is here presented for $\mathrm{Sn}-10 \% \mathrm{~Pb}$ alloy for thermo-physical properties listed in Table 1. The test case is defined for a rectangular domain which is schematically represented in Figure 1. The size of the domain is $10 \mathrm{~cm} \times 6 \mathrm{~cm}$. Due to the symmetry, the computational domain is reduced to 5 $\mathrm{cm} \times 6 \mathrm{~cm}$.

Table 1. Thermo-physical properties of $\mathrm{Sn}-10 \% \mathrm{~Pb}$ and process parameters.

\begin{tabular}{|c|c|c|c|}
\hline Property & Symbol & Value & Unit \\
\hline Density & $\rho$ & $7.00 \mathrm{e}+03$ & $\mathrm{~kg} / \mathrm{m}^{3}$ \\
\hline Specific heat & $c_{p}$ & $2.60 \mathrm{e}+02$ & $\mathrm{~J} / \mathrm{kgK}$ \\
\hline Thermal conductivity & $\lambda$ & $5.50 \mathrm{e}+01$ & $\mathrm{~W} / \mathrm{mK}$ \\
\hline Latent heat of fusion & $L$ & $6.10 \mathrm{e}+04$ & $\mathrm{~J} / \mathrm{kg}$ \\
\hline $\begin{array}{l}\text { Liquid dynamic } \\
\text { viscosity }\end{array}$ & $\mu$ & $1.00 \mathrm{e}-03$ & $\mathrm{Pas}$ \\
\hline $\begin{array}{c}\text { Thermal expansion } \\
\text { coefficient }\end{array}$ & $\beta_{T}$ & $6.00 \mathrm{e}-05$ & $\mathrm{~K}^{-1}$ \\
\hline $\begin{array}{c}\text { Solutal expansion } \\
\text { coefficient }\end{array}$ & $\beta_{C}$ & $-5.30 e-03$ & $\%^{-1}$ \\
\hline Reference temperature & $T_{r e f}$ & $2.19 \mathrm{e}+02$ & ${ }^{\circ} \mathrm{C}$ \\
\hline Reference concentration & $C_{r e f}$ & $1.00 \mathrm{e}+01$ & $\%$ \\
\hline Reference density & $\rho_{\text {ref }}$ & $7.00 \mathrm{e}+03$ & $\mathrm{~kg} / \mathrm{m}^{3}$ \\
\hline Permeability constant & $K_{0}$ & $2.34 \mathrm{e}-11$ & $\mathrm{~m}^{2}$ \\
\hline Melting temperature & $T_{f}$ & $2.32 \mathrm{e}+02$ & ${ }^{\circ} \mathrm{C}$ \\
\hline Eutectic temperature & $T_{e}$ & $1.83 \mathrm{e}+02$ & ${ }^{\circ} \mathrm{C}$ \\
\hline Eutectic concentration & $C_{e}$ & $3.81 \mathrm{e}+01$ & $\%$ \\
\hline $\begin{array}{c}\text { Solubility at eutectic } \\
\text { temperature }\end{array}$ & $C_{e S}$ & $2.49 \mathrm{e}+00$ & $\%$ \\
\hline Liquidus slope & $m_{L}$ & $-1.28 \mathrm{e}+00$ & ${ }^{\circ} \mathrm{C} / \%$ \\
\hline Gravity acceleration & $g_{y}$ & $-9.80 \mathrm{e}+00$ & $\mathrm{~m} / \mathrm{s}^{2}$ \\
\hline External temperature & $T_{e x t}$ & 25 & ${ }^{\circ} \mathrm{C}$ \\
\hline Heat transfer coefficient & $q$ & 400 & $\mathrm{~W} / \mathrm{m}^{2} \mathrm{~K}$ \\
\hline $\begin{array}{l}\text { Equilibrium partition } \\
\text { coefficient }\end{array}$ & $k_{p}$ & 0.0656 & l \\
\hline
\end{tabular}

\subsection{Mathematical model}

\subsubsection{Governing equations}

The problem is described by a set of coupled partial differential equations describing energy and solute transport, and incompressible Newtonian fluid flow with Darcy's approximation for porous media. The microscopic transport is considered through coupling of the solute transport equation and Eutectic phase diagram.
Kozeny-Carman relation is used to account for the porosity of solidifying fluid and Boussinesq approximation is applied to account for the buoyancy forces.

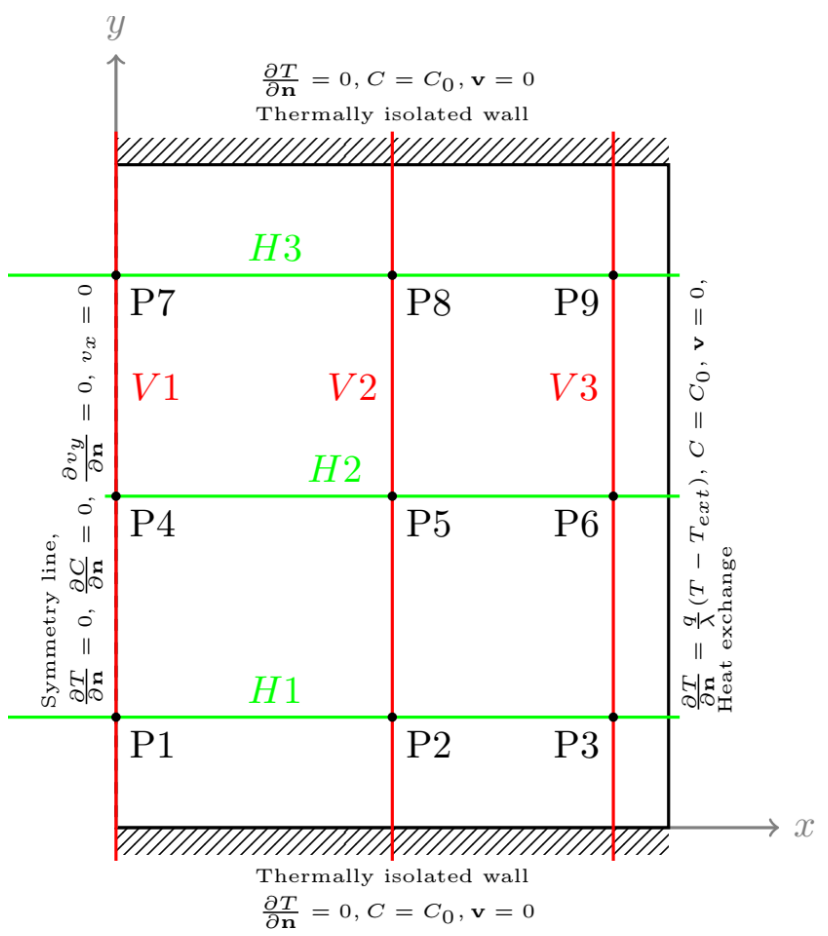

Figure 1. Schematic representation of the computational domain.

$$
\begin{gathered}
\nabla \cdot \mathbf{v}=0 \\
\rho \frac{\partial \mathbf{v}}{\partial t}+\frac{1}{f_{L}} \rho \mathbf{v} \nabla \mathbf{v}=-f_{L} \nabla P+\mu \nabla^{2} \mathbf{v}-\frac{f_{L} \mu}{K} \mathbf{v}+f_{L} \mathbf{b} \\
\rho \frac{\partial h}{\partial t}+\rho \mathbf{v} \cdot \nabla h_{L}=\lambda \nabla^{2} T \\
\frac{\partial C}{\partial t}+\mathbf{v} \cdot \nabla C_{L}=0
\end{gathered}
$$

The liquid velocity $\mathbf{v}=f_{L} \mathbf{v}_{L}$ depends on liquid fraction $f_{L}$ and intrinsic liquid velocity $\mathbf{v}_{L} \cdot \rho$ represents density, $P$ stands for pressure, $\mu$ for viscosity and $K=K_{0} f_{L}^{3} /\left(1-f_{L}\right)^{2}$ for permeability, where $K_{0}$ is permeability constant. The buoyancy term $\mathbf{b}=\rho_{\text {ref }}\left[1-\beta_{T}\left(T-T_{\text {ref }}\right)-\beta_{C}\left(C-C_{r e f}\right)\right] \mathbf{g}$ depends on thermal expansion coefficient $\beta_{T}$, solutal expansion coefficient $\beta_{C}$, temperature $T$, concentration $C$, reference values of temperature $T_{\text {ref }}$, density $\rho_{\text {ref }}$ and concentration $C_{r e f}$, and gravitational acceleration $\mathbf{g}$. Liquidus temperature $T_{L}=T_{F}+m_{L} C_{L}$ is defined as a sum of fusion temperature of the pure solvent $T_{F}$ and a product of liquidus slope $m_{L}$ and liquid concentration $C_{L}$, whereas concentration is defined as 
$C=\left[f_{L}+\left(1-f_{L}\right) k_{p}\right] C_{L}$, where $k_{p} \quad$ is equilibrium partition coefficient. The enthalpy $h=c_{p} T+f_{L} L$ depends on specific heat at constant pressure $c_{p}, T, f_{L}$, and latent heat of fusion $L . \lambda$ is thermal conductivity and $t$ is time.

The pressure-velocity coupling is performed by a fractional step method, where the intermediate velocity is calculated by omitting the pressure correction term. The pressure is then calculated from the Poisson's equation and the velocity is then incremented by a pressure gradient term in order to obtain the divergence-free velocity in the next time step.

\subsubsection{Boundary and initial conditions}

The boundary conditions (Figure 1) at the symmetry line are

$$
\frac{\partial T}{\partial \mathbf{n}}=0, \frac{\partial C}{\partial \mathbf{n}}=0, \frac{\partial v_{y}}{\partial \mathbf{n}}=0, v_{x}=0
$$

At the thermally isolated walls, the boundary conditions are

$$
\frac{\partial T}{\partial \mathbf{n}}=0, C=C_{0}, \mathbf{v}=0
$$

and at the heat exchange wall, the boundary conditions are

$$
\frac{\partial T}{\partial \mathbf{n}}=\frac{q}{\lambda}\left(T-T_{e x t}\right), C=C_{0}, \mathbf{v}=0
$$

The initial conditions in the whole domain and on the boundaries are

$$
\mathbf{v}(t=0)=0, T(t=0)=T_{r e f}, C(t=0)=C_{0} .
$$

\subsection{Meshless solution procedure}

The main challenge addressed in this paper is the application of a meshless approach for the solution of macrosegregation modelling of mesosegregates. The DAM is used for spatial approximation and Euler time stepping scheme is used for time integration.

The global computational domain is divided into local subdomains in such a way that each of the computational nodes $k$ has its own subdomain $(k)$. Each subdomain has $n$ computational nodes. The number of nodes in the subdomain must be at least equal to the size of a polynomial basis $m$. In the present paper, the second order polynomial basis is used $(m=6)$ and each of the subdomains has 13 nodes $(n=13)$. For each of the subdomains, a smooth and differentiable approximation function

$$
\hat{f}^{k}(\mathbf{x})=\mathbf{p}\left(\mathbf{x}, \mathbf{x}_{k}\right) \boldsymbol{\alpha}^{k}=\sum_{j=1}^{m} p_{j}\left(\mathbf{x}, \mathbf{x}_{k}\right) \alpha_{j}^{k}
$$

is obtained. The $\mathbf{p}\left(\mathbf{x}, \mathbf{x}_{k}\right)$ is the second order polynomial basis vector for subdomain $k$ with central computational point $\mathbf{x}_{k}$ and $\boldsymbol{\alpha}^{k}$ vector of coefficients. In order to obtain the approximation coefficient, the following expression has to be minimized

$$
J^{k}=\sum_{i=1}^{m} \theta\left(\mathbf{x}_{i}, \mathbf{x}_{k}\right)\left[f\left(\mathbf{x}_{\mathbf{i}}\right)-\hat{f}^{k}\left(\mathbf{x}_{i}\right)\right]^{2} .
$$

The $f\left(\mathbf{x}_{i}\right)$ are the exact values in point $\mathbf{x}_{i}$ and the $\theta\left(\mathbf{x}_{i}, \mathbf{x}_{k}\right)=\exp \left(-c r_{i}^{2} / h^{2}\right)$ is Gaussian weight function, with $r_{i}$ representing the Euclidian distance between points $\mathbf{x}_{i}$ and $\mathbf{x}_{k}, c=5$ standing for free parameter of the weight function and $h=\max \left(r_{i}\right)$ is a scaling factor. Due to the dominance of the convection term, an upwind scheme is implemented in the DAM weight function [15].

\section{Numerical example}

The code for the present example is implemented in FORTRAN 2008. Intel Visual Fortran compiler (XE14) is used to compile a 64-bit executable. The examples are run on 6 parallel threads on an Intel Xenon at $2 \mathrm{GHz}$. It takes about $17 \mathrm{~h}$ to calculate the present example. The calculations are performed on 151 x 181 node arrangement with time step $\Delta t=1 \cdot 10^{-3} \mathrm{~s}$.

\section{Results and discussions}

The present paper solves the solidification of $\mathrm{Sn}-10 \% \mathrm{~Pb}$ alloy defined by process parameters presented in Table 1 . The results are compared to the results obtained by local radial basis functions colocation method (LRBFCM) [14] and are presented for representative evaluation points $\mathrm{P} 1$ to P9 listed in Table 2. The horizontal and vertical crosssections were observed for three different horizontal (H1 to H3) and vertical (V1 to V3) lines, the details of which are listed in Table 3. The upwinding factor is set to 1 , which means that the calculation point is moved at most halfway in the upwind direction. At the end of each time iteration, the smoothing stage described in [16] is performed and the smoothing factors, which are one of the free parameters of the model are set to $f_{c}=1.0$ for velocity, and $f_{c}=0.025\left(1-f\left(t^{\prime}\right)\right)$ for concentration. The smoothing factor for concentration is applied with a delay

$$
f\left(t^{\prime}\right)=\left\{\begin{array}{c}
1 ; \quad t^{\prime}>1 \\
t^{\prime 2}\left(6-t^{\prime}\left(8-3 t^{\prime}\right)\right) ; \quad t^{\prime}<0 ; t^{\prime}=\frac{t}{\tau} \\
0 ; \quad 1>t^{\prime}>0
\end{array}\right.
$$

where ramp factor is $\tau=0.25$.

Figures 2a-f represent time development of concentration and velocity throughout the solidification process. The thermo-solutal convection starts as a consequence of the cooling of the liquid due to the heat exchange at the outer wall. Thus the flow in the clockwise direction is formed. As the temperature drops 
below the liquidus temperature, the solidification starts. The solute-rich liquid is carried by the flow and thus the solute-poor region is formed at the top of the cavity (Figures 2b-f) and the solute-rich area is gradually formed at the bottom (Figures $2 \mathrm{~b}-\mathrm{f}$ ) and at the centre of the cavity (Figures 2e-f). The mesosegregates form in the shape of channel-like anomalies in the mushy zone region (Figures 2d-f). The instabilities that are the driving force behind the formation of these channels propagate the

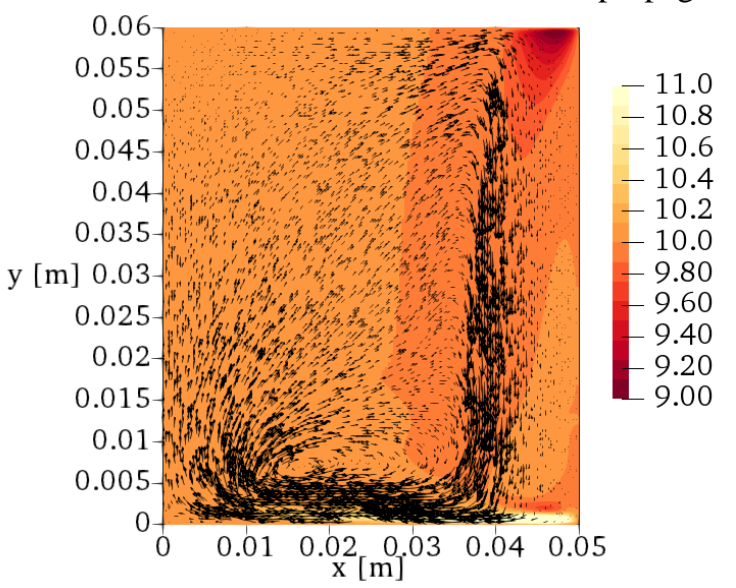

a) $t=10 \mathrm{~s}$

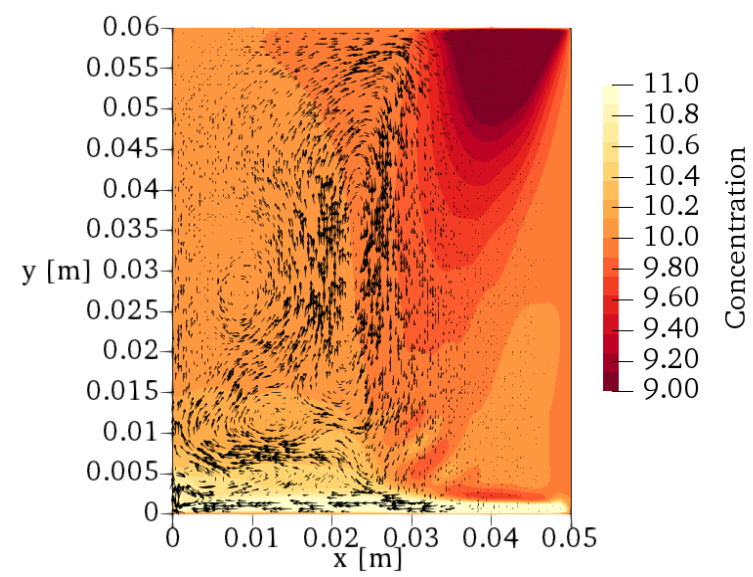

c) $t=40 \mathrm{~s}$.

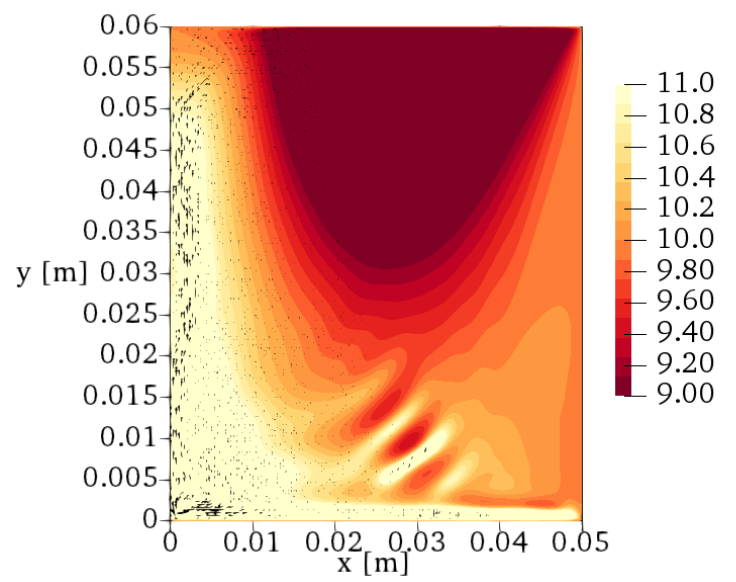

e) $t=120 \mathrm{~s}$ flow and consequently the segregation, thus forming the patches with alternating areas of positive and negative segregation (Figures 2d-f). Although the mechanism behind the formation of these channels is not well known, the numerical instabilities are not the reason for their appearance, as these mesosegragate formations have also been observed experimentally [13].

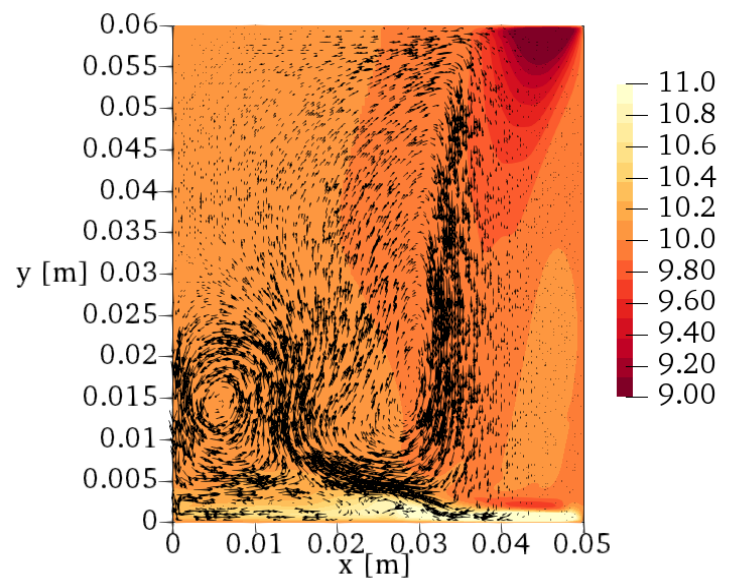

b) $t=20 \mathrm{~s}$.

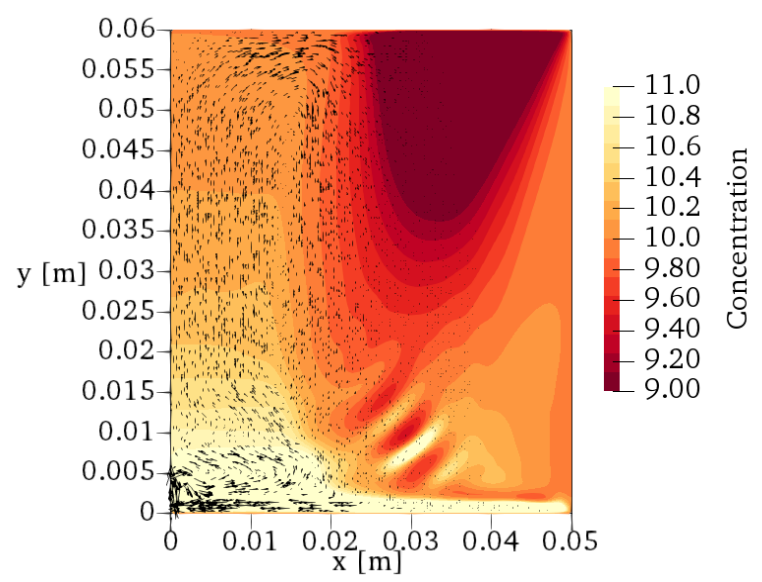

d) $t=80 \mathrm{~s}$

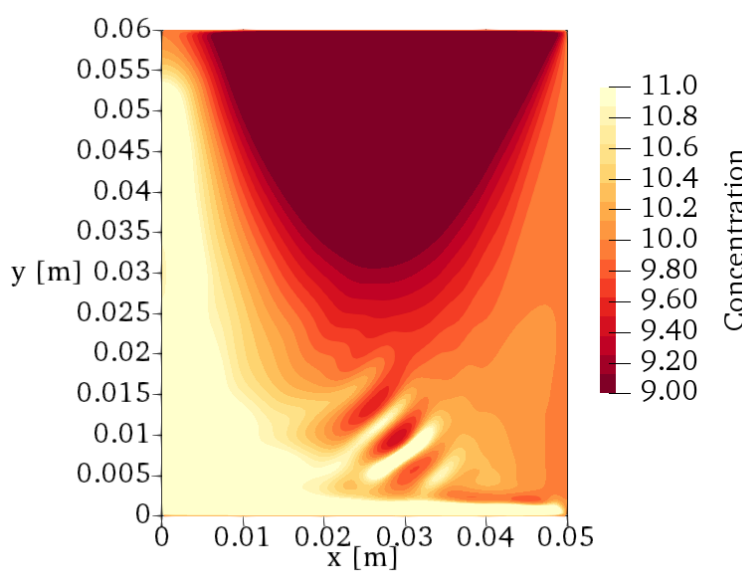

f) $t=600 \mathrm{~s}$

Figure 2. Concentration [\%] and velocity vector at different times. 
The results have also been analysed for reference points defined in Table 2 and cross-sections defined in Table 3. Figure 3 presents concentration profiles for horizontal cross-sections. The profiles correspond to the final time step of the computational example (Figure $2 \mathrm{f}$ ). The H3 line shows a smooth distribution of concentration in the top part of the cavity. The Sn concentration is the highest at the centre of the cavity and at the outer (heat exchange) wall and the lowest in the middle of the computational domain. The $\mathrm{H} 1$ line shows horizontal cross-section close to the bottom of the cavity and shows a perturbation at the middle of the computational domain. The perturbation corresponds to the segregation channels (mesosegregates). Figure 4 presents the concentration of $\mathrm{Sn}$ for vertical cross-sections at V1 to V3. The peak in blue line (V3) corresponds to the increased concentration at the bottom of the cavity close to the outer wall. The concentration of Sn then gradually drops toward the top of the cavity. The V1 line presents the concentration at the centre of the cavity where the area with the positive $\mathrm{Sn}$ concentration is formed. The slight decrease in the middle is due to the velocity recirculation pattern in the final stages of solidification as can be seen in Figure 2e. The pattern is formed due to the temperature, which in term causes the change in liquid fraction. The liquid consequently flows around this part and thus the velocity in the totally liquid part of the fluid is increased in contrast to the velocity in the partially solidified fluid, which is decreased. In both cases (horizontal and vertical), the $\mathrm{H} 2$ and $\mathrm{V} 2$ lines present the cross-sections in the middle of the horizontal and vertical domains.

Table 2. Definition of benchmark control points defined for SMACS benchmark test case.

\begin{tabular}{|c|c|}
\hline Point & Coordinates $(\mathbf{x}[\mathbf{m}], \mathbf{y}[\mathbf{m}])$ \\
\hline P1 & $(0.0201)$ \\
\hline P2 & $(0.045,0.01)$ \\
\hline P3 & $(0.0,0.03)$ \\
\hline P4 & $(0.025,0.03)$ \\
\hline P5 & $(0.045,0.03)$ \\
\hline P6 & $(0.0,0.05)$ \\
\hline P7 & $(0.025,0.05)$ \\
\hline P8 & $(0.045,0.05)$ \\
\hline P9 &
\end{tabular}

The results for temperature, liquid fraction and concentration are listed in Table 4 for all representative points $\mathrm{P} 1$ to $\mathrm{P} 9$. The results are listed at $t=570 \mathrm{~s}$, when the whole computational domain is solidified. It should be noted here, that in comparison with [14], the results of the present study show lower positive segregation in the central area than the results calculated with LRBFCM [14]. The results for V2 and V3 are in good agreement with reference results as are points $\mathrm{P} 2, \mathrm{P} 3, \mathrm{P} 5, \mathrm{P} 6, \mathrm{P} 8$ and P9 that do not lie on the centre line.

Time developments of temperature, liquid fraction and concentration fields are presented in Figures 5 to 7. As already mentioned, the solidification starts once the temperature drops below the liquidus temperature and lasts until it reaches the eutectic temperature $\left(T_{\text {eut }}=183^{\circ} \mathrm{C}\right)$. Figure 5 compares temperature for calculated results (lines) with reference results [14] (crosses) in representative points (P1 to P9). The results are in good agreement.

Table 3. Definition of benchmark control lines defined for SMACS benchmark test case.

\begin{tabular}{|c|c|}
\hline Line & Location $([\mathrm{m}])$ \\
\hline H1 & $\mathrm{y}=0.01$ \\
\hline H2 & $\mathrm{y}=0.03$ \\
\hline H3 & $\mathrm{y}=0.05$ \\
\hline V1 & $\mathrm{x}=0.00$ \\
\hline V2 & $\mathrm{x}=0.025$ \\
\hline V3 & $\mathrm{x}=0.045$ \\
\hline
\end{tabular}

Table 4. Representation of results in representative points at $t=570 \mathrm{~s}$.

\begin{tabular}{|c|c|c|c|}
\hline Point & $T\left[{ }^{\circ} \mathbf{C}\right]$ & $f_{L}$ & $C[\%]$ \\
\hline P1 & 109.30 & 0.00 & 12.76 \\
\hline P2 & 105.91 & 0.00 & 10.81 \\
\hline P3 & 98.49 & 0.00 & 10.43 \\
\hline P4 & 109.30 & 0.00 & 10.72 \\
\hline P5 & 105.90 & 0.00 & 9.17 \\
\hline P6 & 98.49 & 0.00 & 9.97 \\
\hline P7 & 110.29 & 0.00 & 12.29 \\
\hline P8 & 105.91 & 0.00 & 7.77 \\
\hline P9 & 98.49 & 0.00 & 9.28 \\
\hline
\end{tabular}




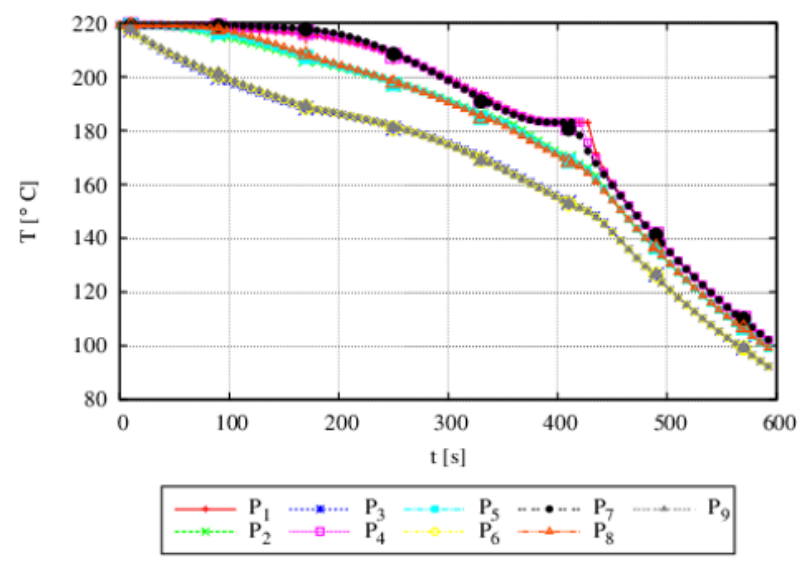

Figure 5. Time development of temperature in representative points P1 to P9. Lines and smaller nodes depict results calculated with DAM and bigger nodes depict results calculated with LRBFCM [14].

The time development of liquid fraction is presented in Figure 6 for calculated results. In the present case, the solidification stops, when the temperature falls below eutectic temperature, which can be clearly seen in Figure 6 , where the liquid fraction drops to zero after reaching the eutectic temperature. The effect of the described model is best observed for points at the centre line (P1, $\mathrm{P} 4$ and $\mathrm{P} 7$ ) and can be also seen on the temperature graph (Figure 5).

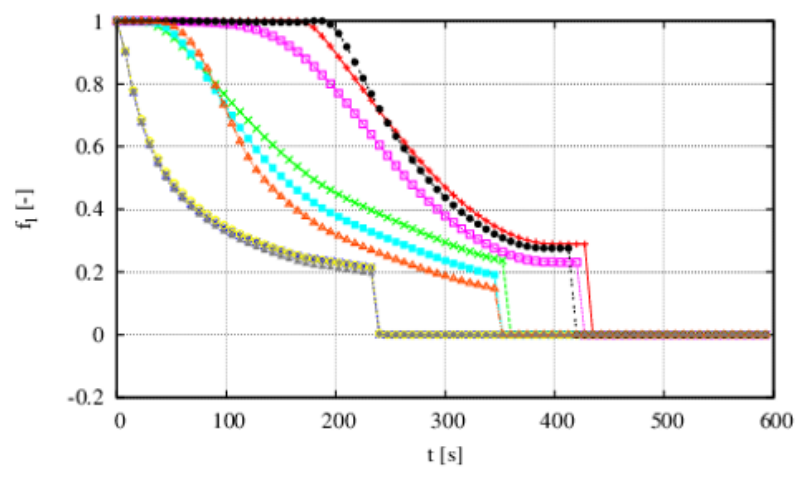

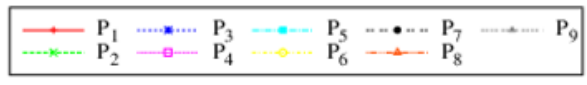

Figure 6. Time development of liquid fraction in representative points $\mathrm{P} 1$ to $\mathrm{P} 9$.

Figure 7 presents the time development of Sn concentration in representative points. The solidification begins when the temperature drops below the liquidus temperature and stops when the eutectic temperature is reached.

Finally, a comparison is made for a uniform and nonuniform node arrangement with randomly positioned nodes [17] and is presented in Figures $2 \mathrm{f}$ (uniform) and 8 (non-uniform). Although the final macrosegregation pattern is similar for both of the cases, there are observable differences in the mesosegregates. The formed channels are much more pronounced in nonuniform grid example, which can be clearly seen in Figures 3 and 4 . The difference might be due to the upwind and smoothing parameters, which might require different values for a non-uniform node arrangement. However, this was not observed in previous simulations of macrosegregation [15] without mesosegregates. The results show that formulation of channels strongly depends on the node density and arrangement. Similar observations were made in [9]. The simulation with various models and methods showed that it is not possible to obtain mesh-converged results.

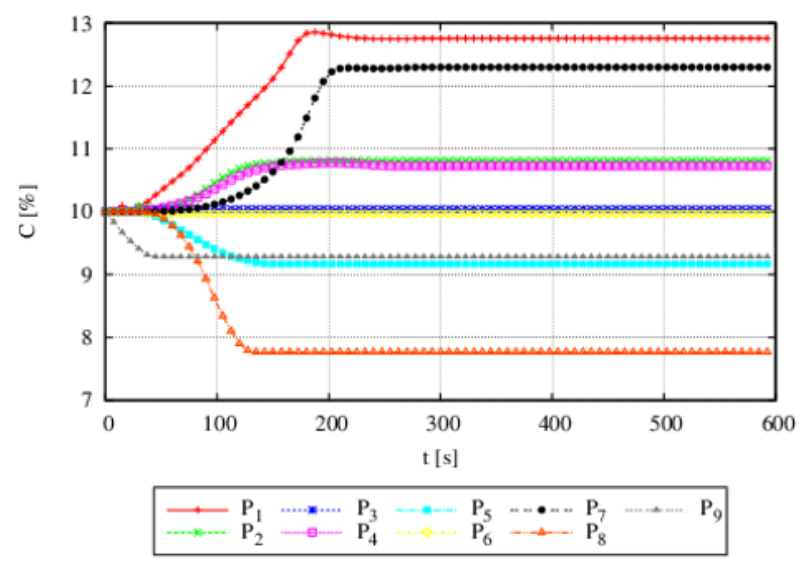

Figure 7. Time development of concentration in representative points $\mathrm{P} 1$ to $\mathrm{P} 9$.

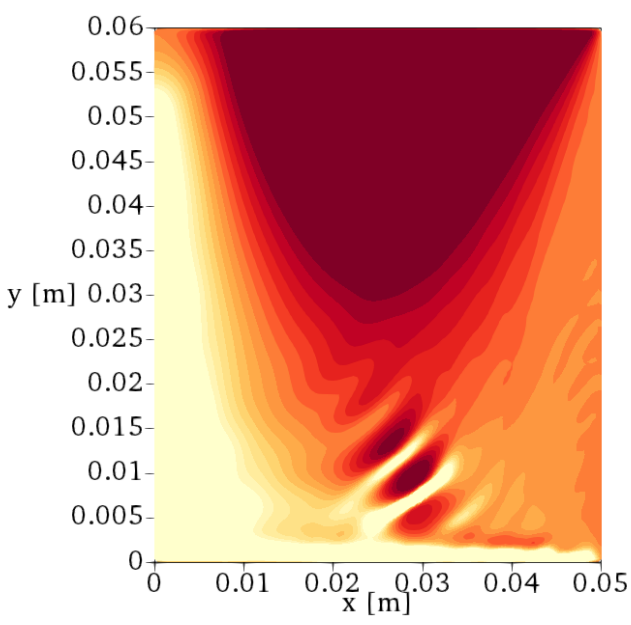

Figure 8. Concentration [\%] and velocity vector for a fully solidified case for a non-uniform node arrangement $(t=600 \mathrm{~s})$.

\section{Conclusions}

The present paper presents a solution of a mathematical model for macrosegregation with mesosegregates in a binary substrate solved by DAM. The problem corresponds to the benchmark example for $\mathrm{Sn}-10 \% \mathrm{~Pb}$ alloy proposed in [12] and the results are compared to those obtained with LRBFCM presented by [14]. The results are in good agreement with reference results.

\section{Acknowledgement}


This work was founded by Slovenian research agency (ARRS) in the framework of basic research project J27384 and program group P2-0162.

\section{References}

1. "Web of Science [v.5.28] - Web of Science Core Collection Result Analysis." [Online]. Available: http://wcs.webofknowledge.com/RA/analyze.do. [Accessed: 10-Apr-2018].

2. E. J. Kansa, CMA, 19, 127 (1990)

3. W. Chen, Meshfree methods for partial differential equations, (Springer, 2003)

4. S. N. Atluri, T. Zhu, Comput. Mech. 22, 117 (1998)

5. G.-R. Liu, Mesh free methods: moving beyond the finite element method. (CRC Press, 2002)

6. B. Šarler and R. Vertnik, CMA, 51, 1269 (2006)

7. C. Prax, H. Sadat, P. Salagnac, Transp. Porous Media, 22, 215 (1996)

8. B. Šarler, R. Vertnik, J. Perko, CMA, 2, 77 (2005)

9. H. Combeau et al., Mater. Sci. Eng., 33 (2012)

10. H. Sadat, N. Dubus, L. Gbahoue, T. Sophy, Numer. Heat Transfer, Part B, 50, 491 (2006)

11. H. Sadat and C. Prax, Int. J. Heat Mass Transfer, 39, 214 (1996)

12. M. Bellet et al., Int. J. Therm. Sci. 48, 2013, (2009)

13. J. Sarazin, A. Hellawell, Metall. Trans., 19, 1861 (1988)

14. G. Kosec, B. Šarler, EABE, 45, 36 (2014)

15. V. Hatić, B. Mavrič, B. Šarler, Int. J. Numer. Methods Heat Fluid Flow, 28, 361 (2018)

16. K. Reuther, B. Sarler, M. Rettenmayr, Int. J. Therm. Sci., 51, 16 (2012)

17. V. Hatić, B. Mavrič, N. Košnik, B. Šarler, Appl.Math. Model., 54, 170 (2018) 